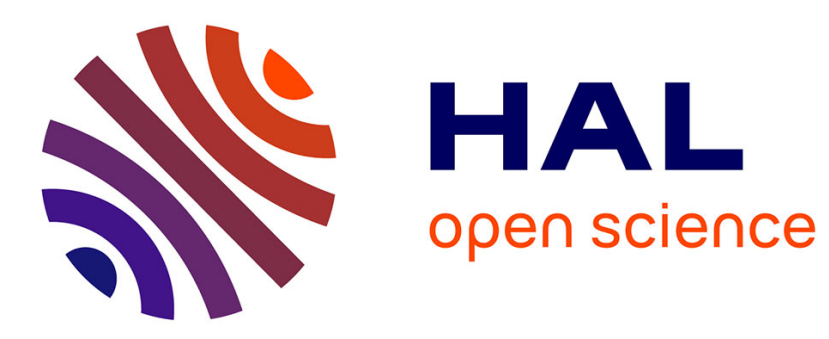

\title{
Episode Rules Mining Algorithm for Distant Event Prediction
}

\author{
Lina Fahed, Armelle Brun, Anne Boyer
}

\section{To cite this version:}

Lina Fahed, Armelle Brun, Anne Boyer. Episode Rules Mining Algorithm for Distant Event Prediction. [Research Report] 2014. hal-01062542

\section{HAL Id: hal-01062542 \\ https://inria.hal.science/hal-01062542}

Submitted on 10 Sep 2014

HAL is a multi-disciplinary open access archive for the deposit and dissemination of scientific research documents, whether they are published or not. The documents may come from teaching and research institutions in France or abroad, or from public or private research centers.
L'archive ouverte pluridisciplinaire HAL, est destinée au dépôt et à la diffusion de documents scientifiques de niveau recherche, publiés ou non, émanant des établissements d'enseignement et de recherche français ou étrangers, des laboratoires publics ou privés. 


\title{
Episode Rules Mining Algorithm for Distant Event Prediction
}

\author{
Lina Fahed ${ }^{1}$, Armelle Brun ${ }^{1}$ and Anne Boyer ${ }^{1}$ \\ ${ }^{1}$ Université de Lorraine - LORIA - Équipe KIWI \\ Campus scientifique, BP 239 54506, Vandoeuvre-lès-Nancy Cedex, France \\ \{lina.fahed, armelle.brun, anne.boyer\}@loria.fr
}

Keywords: Data Mining, Episode Rules Mining, Minimal Rules, Distant Event Prediction

Abstract: This paper focuses on event prediction in an event sequence, where we aim at predicting distant events. We propose an algorithm that mines episode rules, which are minimal and have a consequent temporally distant from the antecedent. As traditional algorithms are not able to mine directly rules with such characteristics, we propose an original way to mine these rules. Our algorithm, which has a complexity similar to that of state of the art algorithms, determines the consequent of an episode rule at an early stage in the mining process, it applies a span constraint on the antecedent and a gap constraint between the antecedent and the consequent. A new confidence measure, the temporal confidence, is proposed, which evaluates the confidence of a rule in relation to the predefined gap. The algorithm is validated on an event sequence of social networks messages. We show that minimal rules with a distant consequent are actually formed and that they can be used to accurately predict distant events.

\section{INTRODUCTION}

The flow of messages posted in blogs and social networks is an important and valuable source of information that can be analyzed, modeled (through the extraction of hidden relationships) and from which information can be predicted, which is the focus of our work. For example, companies may be interested in the prediction of what will be said about them in social networks. Similarly, this prediction can be a way to recommend items. We consider that the sooner an event is predicted, the more useful this prediction is for the company or the person concerned, since this allows to have enough time to act before the occurrence of the event. Predicting distant events is thus the focus of our work.

Temporal data mining is related to the mining of sequential patterns ordered by a given criterion such as time or position (Laxman and Sastry, 2006). Episode mining is the appropriate pattern discovery task related to the case the data is made up of a single long sequence. An episode is a temporal pattern made up of "relatively close" partially ordered items (or events), which often appears throughout the sequence or in a part of it (Mannila et al., 1997). When the order of items is total, the episode is said to be serial. Similarly to the extraction of association rules from itemsets, episode rules can be extracted from episodes to predict events (Cho et al., 2011). The rule mining task is usually decomposed into two subproblems. The first one is the discovery of frequent itemsets or episodes that have a support higher than a predefined threshold. The second one is the generation of rules from those frequent itemsets or episodes, with the constraint of a minimal confidence threshold (Agrawal et al., 1993). In general, rules are generated by considering some items in the itemset (or the last items in the episode) as the consequent of the rule, and the rest of the items as the antecedent. Since the second sub-problem is quite straightforward, most of the researches focus on the first one: the extraction of itemsets or episodes.

Episode and episode rules mining are used in many areas, such as telecommunication alarm management (Mannila et al., 1997), intrusion detection (Luo and Bridges, 2000), discovery of relation between financial events ( $\mathrm{Ng}$ and $\mathrm{Fu}, 2003$ ), etc.

Our goal is to reliably predict events that will occur after a predetermined temporal distance, in order to have enough time to act before the occurrence of events. Therefore, serial episode rules with a consequent distant from the antecedent will be mined. Traditional episode rules mining algorithms form episode rules with a consequent close to the antecedent. To mine rules with a distant consequent, these algorithms have to perform a post-processing step: the extracted 
rules are filtered to keep only rules with a consequent that may occur far from the antecedent. This postprocessing is not only time consuming, but also the time required for mining rules which will be filtered out is useless. Thus, we propose a new algorithm for mining serial episode rules, with a consequent temporally distant from the antecedent, to predict distant events, and with a small antecedent (in number of events and in time), to be able to predict events as soon as possible. This algorithm has a complexity equal to that of traditional algorithms.

An example of a required rule is presented below (from a sequence of annotated messages of blogs about finance issues, where each event includes a sentiment polarity): $R$ : (interest rate, neutral) (credit, negative) (waiting, neutral) $\rightarrow$ (concurrence, negative); the antecedent occurs within 5 days, the gap between the antecedent and the consequent is 15 days.

The rest of this paper is organized as follows: section 2 presents related works about episode rules mining. Our algorithm is introduced in section 3 , followed by experimental results in section 4 . We conclude and provide some perspectives in section 5 .

\section{RELATED WORKS}

We first start by introducing few concepts. Let $I=\left\{i_{1}, i_{2}, \ldots, i_{m}\right\}$ be a finite set of items. $I_{t}$ is the set of items that occur at a timestamp $t$ referred to as an event. An event sequence $S$ is an ordered list of events, $S=<\left(t_{1}, I_{t_{1}}\right),\left(t_{2}, I_{t_{2}}\right), \ldots,\left(t_{n}, I_{t_{n}}\right)>$ with $t_{1}<$ $t_{2}<\ldots<t_{n}$, (see Figure 1). The serial episode $P=<$ $p_{1}, p_{2}, \ldots, p_{k}>$ on $I^{k}$ is an ordered list of events. Its support, denoted to as $\sup p(P)$, represents the number of occurrences of $P$, according to a frequency measure. $P$ is said to be a frequent episode if $\operatorname{supp}(P) \geq$ minsupp where minsupp is the predefined minimal threshold. An occurrence window of the episode $P$ is a segment $\left\langle I_{t_{s}}, \ldots, I_{t_{e}}\right\rangle$ of the sequence, denoted as $O W\left(S, t_{s}, t_{e}\right)$ which starts at timestamp $t_{S}$ and ends at timestamp $t_{e}$, where $P \subseteq<I_{t_{s}}, \ldots, I_{t_{e}}>, p_{1} \subseteq I_{t_{s}}$ and $p_{k} \subseteq I_{t_{e}}$. It represents the interval that bounds the episode. Let $P, Q$ be two episodes. An episode rule $R: P \rightarrow Q$ means that $Q$ appears after $P$. The confidence of this episode rule is the probability to find $Q$ after $P: \operatorname{con} f(P \rightarrow Q)=\operatorname{supp}(P . Q) / \operatorname{supp}(P)$. The rule is said to be confident if its confidence exceeds a predefined threshold minconf.

Winepi and Minepi are seminal episode mining algorithms (Mannila et al., 1997), and are the basis of many other algorithms. To extract episodes, both algorithms start by extracting 1-tuple episodes (made up of one item), then iteratively seek larger ones by merging items on their right side. This approach is the one used by many other algorithms (Laxman et al. 2007) (Huang and Chang, 2008).

In the frequent itemsets mining task (which includes episode mining), it is suggested that antimonotonicity is a common property that has to be respected by any frequency measure (Agrawal et al., 1993). Several frequency measures for episode mining have been proposed. In (Mannila et al., 1997), window-based and minimal occurrence-based frequency measures have been introduced through both Winepi and Minepi. Winepi evaluates the frequency of an episode as the number of windows of length $w$ that contain the episode. Minepi evaluates the frequency of an episode as the number of minimal windows that contain the episode. A minimal window is a window such that no sub-window contains the episode. The non-overlapped occurrencebased frequency measure is proposed (Laxman et al. 2007), where two occurrences of an episode are nonoverlapped if no item of one occurrence appears in between items of the other. It is shown that the nonoverlapped occurrence-based algorithms are much more efficient in terms of space and time needed. When mining serial episodes, additional constraints on the episodes may be imposed. Such as the span constraint (Achar et al., 2013), that imposes an upper bound (of distance or time) between the first and last event in the occurrence of an episode. This constraint has been mainly introduced for complexity reasons. Another constraint is the gap constraint (Méger and Rigotti, 2004), which imposes an upper bound between successive events in the occurrence of an episode. If the extracted serial episodes have to represent causative chains then such constraints are important.

Traditional episode rules mining algorithms construct episode rules with a large antecedent (made up of many events) (Pasquier et al., 1999). Discovering rules with a small antecedent was introduced for association rules, called "minimal association rules discovery" (Rahal et al., 2004). Minimal rules are considered as sufficient (as no new knowledge is given by larger ones). The constraint is that the consequents are fixed in advance by the user. Minimal rules have also been studied with the aim to reduce time and space complexity of the mining task, as well as to avoid redundancy in the resulting set of rules (Neeraj and Swati, 2012). These works focus on association rules; recall we want to form episode rules.

Mining episodes in an event sequence is a task which has received much attention (Gan and Dai, 2011). In an event sequence each data element may contain several items (an event). In (Huang and 


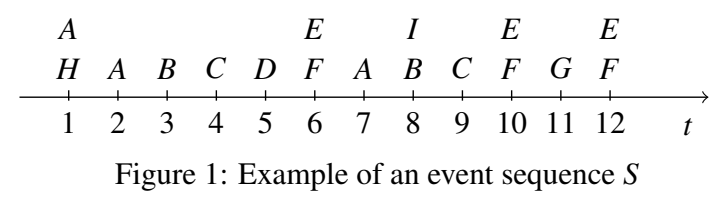

Chang, 2008), an algorithm Emma, is presented, where the event sequence is encoded with frequent itemsets then serial episodes are mined. In (Gan and Dai, 2011), episodes are first extracted, then nonderivable episodes rules are formed (where no rule can be derived from another).

\section{THE PROPOSED ALGORITHM}

\subsection{Principle}

Our goal is to form episode rules that can also be used to efficiently predict distant events.

To achieve this goal, the episode rules formed have to hold the characteristic that the consequent is temporally distant from the antecedent. Traditional algorithms are not designed to form such rules. Recall that they first form episodes from left to right by iteratively appending events temporally close to the episode being formed (the minimal occurrence), in the limit of the predefined span. Then, the episode rule is built by considering the last element(s) of the episode as consequent of the rule. In addition, when forming these episodes, it is impossible to know if the event being appended will be part of the consequent or not, so it is impossible to constrain its distance to other events while forming the episode. The only way to mine rules with a consequent distant from the antecedent is to mine all rules and then filter the occurrences that do respect this distance. Due to the limited span, this distance cannot be large.Thus, we propose to mine episode rules without any episode mining phase. To be able to constrain the distance between the antecedent and the consequent, we propose to determine the consequent early in the mining process. We think that, by determining the consequent at an early stage, the occurrence windows of an episode rule will be filtered early, thus the search space will be pruned, and no post-processing is required.

We aim also at predicting events early. We assume that the more the antecedent of a rule is small in number of events and in time, the earliest it ends, and the earliest the consequent can be predicted. Therefore, we propose to extract episode rules that have an additional characteristic: an antecedent as small as possible (in number of events and in time), which we call "minimal episode rules". For that, we apply the traditional minimal occurrence-based frequency measure.

The required characteristics force us to propose an approach totally different from traditional ones. We propose an episode rules mining algorithm, where the prefix (the first event) of a rule is first fixed. Then, the consequent is determined to constrain its distance from the prefix. Finally, the antecedent is completed.

Before explaining our algorithm, we present new concepts on which our algorithm relies.

Sub-windows of $\operatorname{Win}\left(S, t_{s}, w\right)$ : Let $\operatorname{Win}\left(S, t_{s}, w\right)$ be a window in the sequence $\mathrm{S}$ of length $w$ that starts at $t_{s}$, with its first element containing the prefix of an episode rule (the first event) to be built. In order to mine episode rules with a distant consequent and a minimal antecedent, we propose to split this window into three sub-windows as follows (see Figure 2):

$W_{\text {in }}$ begin is a segment of $\operatorname{Win}\left(S, t_{s}, w\right)$ of length $w_{\text {begin }}<w$, starting at $t_{s}$. Win begin $_{\text {be }}$ can be viewed as an expiry time for the antecedent of an episode rule. It represents the span of the antecedent of an episode rule to guarantee that the antecedent occurs within a determined time.

$W_{\text {in }}$ end is a segment of $\operatorname{Win}\left(S, t_{s}, w\right)$ of length $w_{\text {end }}<w$, that ends at $t_{s}+w$. Win end represents the time window of occurrence of the consequent.

Win $_{\text {between }}$ is the remaining sub-window of length $w_{\text {between }}$, in which neither the antecedent nor the con-

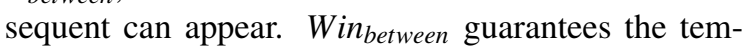
poral distance between the antecedent and the consequent of an episode rule. It represents a minimal gap between the antecedent and the consequent to guarantee that the consequent is far from the antecedent.

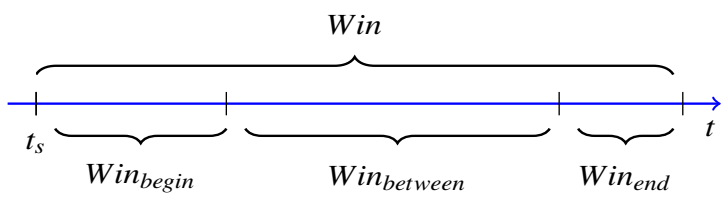

Figure 2: Sub-windows of $\operatorname{Win}\left(S, t_{s}, w\right)$

\subsection{Steps of the algorithm}

We present now the different steps of our algorithm.

\subsubsection{Initialization}

Our algorithm starts by an initialization phase, which reads the event sequence to extract all frequent events and their associated occurrence timestamps. An event represents a 1-tuple episode and will be denoted as $P$. Table 1 presents the list of 1-tuple episodes of the sequence $S$ (Figure 1) and their associated occurrence windows for minsupp $=2$ (see Figure 3 line 2). 
Table 1: 1-tuple episodes of $S$.

\begin{tabular}{cc}
\hline 1-tuple episode $p$ & List of occurrence windows \\
\hline A & {$[1,1],[2,2],[7,7]$} \\
B & {$[3,3],[8,8]$} \\
C & {$[4,4],[9,9]$} \\
E & {$[6,6],[10,10],[12,12]$} \\
F & {$[6,6],[10,10],[12,12]$} \\
EF & {$[6,6],[10,10],[12,12]$} \\
\hline
\end{tabular}

\subsubsection{Prefix identification}

Episode rules are built iteratively by first fixing the prefix (the first event of the antecedent). The antecedent is denoted as ant. In the encoded sequence, each 1-tuple episode $p$ is viewed as a prefix of the antecedent of an episode rule to be built. Once the prefix of an episode rule $R$ is fixed, its occurrence windows $O W\left(S, t_{s}, t_{e}\right)$ are known. For example, let minsupp $=2, A$ can be considered as a prefix of an episode rule. The list of occurrence windows of $A=$ $([1,1],[2,2],[7,7])$ (see Table 1).

\subsubsection{Consequent identification}

A candidate consequent of an antecedent ant (here, ant corresponds to a unique element, the prefix) is chosen in the windows $\operatorname{Win}\left(S, t_{s}, w\right)$ where: ant $\subseteq I_{t_{s}}$. Recall we want to form episode rules with a consequent as far as possible from the antecedent. Thus, the candidate consequents are not searched in the entire window, they are searched only in $W_{i n}$ end where the farthest candidates are. We construct $\mathcal{P}_{\text {end }}($ ant $)$, the ordered list of 1-tuple episodes that occur frequently in $\operatorname{Win}_{\text {end }}$ from $\operatorname{Win}\left(S, t_{s}, w\right)$ that contains ant.

Let $p_{j} \in \mathcal{P}_{\text {end }}($ ant $)$ be a candidate consequent of ant. The episode rule $R:$ ant $\rightarrow p_{j}$ is formed and its support is computed (see Figure 3 line 7). At this stage, the occurrence windows of the episode rule $R$ are filtered to get minimal occurrences as well as to preserve the anti-monotonicity property. This filtering is done by counting only once the occurrence windows containing the same occurrence of the consequent $p_{j}$. For example, let $w_{\text {begin }}=2, w_{\text {end }}=2$ and $w=6$. The episode rule $R: A \rightarrow E$ has three occurrence windows: $([1,6],[2,6],[7,12])$. In the two first occurrence windows, the consequent $E$ is a common 1 -tuple episode which occurs at timestamp $t_{6}$. Therefore, we consider only the interval $[2,6]$. However, all occurrence windows are kept in memory, to be used to complete the antecedent. This enables not losing any of the interesting episode rules which could be missed if we kept only the minimal occurrences in memory.

Next, we compute the support of the corresponding episode rules (see Figure 3 line 7).
We define the support of a rule $P \rightarrow Q$, referred to as $\operatorname{supp}_{\text {end }}(P . Q)$, as the number of minimal occurrence windows computed above. It is different from the traditional $\operatorname{supp}(P . Q)$ as it considers only occurrence windows where $P$ occurs in $W_{i n}$ begin and $Q$ occurs in $W_{i n}$ end .

If $R:$ ant $\rightarrow p_{j}$ is not frequent, we consider that $p_{j}$ cannot be a consequent of ant. This iteration is stopped and the rule is discarded. There is no need to complete the antecedent of the rule $R$, as whatever the events that complete the antecedent are, the resulting rule will not be frequent. The algorithm will iterate on another consequent. If $R: a n t \rightarrow p_{j}$ is frequent, its confidence is computed. its confidence is computed.

We define the confidence of a rule $P \rightarrow Q$ (see Equation (1)) as the probability that the consequent occurs in $W_{i n}$ end , given that $P$ appears in $W_{i n}$ begin .

$$
\operatorname{conf}(P \rightarrow Q)=\frac{\operatorname{supp}_{\text {end }}(P \cdot Q)}{\operatorname{supp}(P)}
$$

If the rule $R:$ ant $\rightarrow p_{j}$ is confident, this rule is added to the set of rules formed by the algorithm. It is minimal and has a consequent far from the antecedent; it fulfills our goal. If the rule is frequent but not confident, the antecedent of the rule $R:$ ant $\rightarrow p_{j}$ is completed (as in the next subsection).

For example, let $w=6, w_{\text {begin }}=2$ and $w_{\text {end }}=2$. For the episode rule $R$ with prefix $A . \quad \mathcal{P}_{\text {end }}(A)=$ $[E, F, E F, A]$. We first try to construct the episode $R$ with the consequent $E$. Thus, for $R: A \rightarrow$ $E, \operatorname{supp}(R)=2$ and $\operatorname{conf}(R)=2 / 3=0.67$. For minsupp $=2$ and minconf $=0.7, R$ is frequent but not confident, so its antecedent has to be completed.

\subsubsection{Antecedent completion}

In this step, the antecedent, referred to as ant, is iteratively completed with 1-tuple episodes, placed on its right side in the limit of the predefined sub-window $W_{i_{\text {begin }}}$. At the first iteration: ant is a unique element (the prefix) (see Figure 3 line 15). Recall that we aim at forming rules having the last event of the antecedent as far as possible from the consequent, so as close as possible of the prefix. Thus, we construct $\mathcal{P}_{\text {begin }}($ ant $)$, the ordered list of 1-tuple episodes that occur frequently after ant in the windows that starts with it: the 1-tuple episodes that occur in $W_{\text {in }}$ begin.

Similarly to the consequent identification step, the occurrence windows of the episode rule $R$ are filtered to get minimal occurrences and to preserve the anti-monotonicity property. In addition, we apply the same support, confidence verifications as for the consequent identification.

To speed up the episode rules mining process we use a heuristic. We propose to order the list of candi- 
dates $\mathcal{P}_{\text {begin }}$ (ant) in descending order of the number of $W_{\text {in }_{\text {begin }}}$ in which the candidates appear. We assume that this number is highly correlated with the support of the corresponding episode rules. So, in the traversal of this list, when we observe that candidates tend to form infrequent episode rules (several consecutive 1-tuple episodes lead to infrequent episode rules), we stop the traversal. We consider that the remaining candidates in this list will lead to infrequent episode rules. This heuristic is used to reduce the number of iterations. Although this heuristic may discard interesting rules, it allows to reduce the iterations thus to increase the size of the span of the rule (Win).

For example, let minsupp $=2$ and mincon $f=0.7$, for $R: A \rightarrow E, \mathcal{P}_{\text {begin }}(A)=[B, C, A]$. The antecedent of $R$ is completed with $B$ and forms the episode rule $R$ : $A, B \rightarrow E$. Thus, $\operatorname{supp}(R)=2$ and $\operatorname{conf}(R)=2 / 2=$ 1. The episode rule $\mathrm{R}$ is now confident, the phase of completing its antecedent is stopped.

\subsection{Temporal confidence}

Win between $_{\text {has }}$ been introduced so as to guarantee that the consequent of an episode rule occurs in $W_{i n}$ end, after a $w_{\text {begin }}+w_{\text {between }}$ temporal distance from the prefix of the episode rule. However, given an occurrence of the antecedent, the consequent may also occur closer to the antecedent, in the window Win $_{\text {between }}$, which should affect the confidence of the rule. This information may be important in some applications. In the example of social networks, the prediction of a negative event allows the company to act so as to prevent its occurrence. So, it is important to mine rules with a consequent that never occurs in $W_{i n}$ between . Indeed, predicting a consequent at a given distance, which may appear closer is useless, even dangerous. Consequently, we have to take into consideration the occurrence of the consequent in $W_{\text {in }}$ between. We introduce a new measure, the temporal confidence, which represents the probability that the consequent occurs in Win $_{\text {end }}$ and only in it. For an episode rule $R: P \rightarrow Q$, this measure takes into account the support of $P . Q$ when $Q$ occurs only in $W_{i n}$ end (and not in $W_{\text {in }}$ between $)$, denoted as $\operatorname{supp}_{\text {end }}(P . Q)$. The temporal confidence is defined as follows:

$$
\operatorname{conf}_{t}(P \rightarrow Q)=\frac{\operatorname{supp}_{\text {end }}(P \cdot Q)}{\operatorname{supp}(P . Q)}
$$

$\operatorname{con} f_{t}(R)=1$ if for each occurrence of the consequent of $R$ in $W i n_{\text {end }}$, no occurrence of the consequent is found in $W i n_{\text {between }}$. The temporal confidence of rules from the previous section is computed and the rules with a temporal confidence above mincon $f_{t}$ are kept. For example, let $w=6, w_{\text {begin }}=2$ and $w_{\text {end }}=2$, the temporal confidence of the frequent confident episode rule $R: A \rightarrow E$ depends on the occurrences of $E$ in $W$ in $_{\text {between }}$ which is equal to 1 ( $E$ appears in the timestamp $t_{10}$ in Win $\left._{\text {between }}\right)$. Thus, $\operatorname{conf}_{t}(R: A \rightarrow E)=$ $1 / 2=0.5$. For mincon $f_{t}=0.5, R$ is temporally confident and is a rule formed by our algorithm.

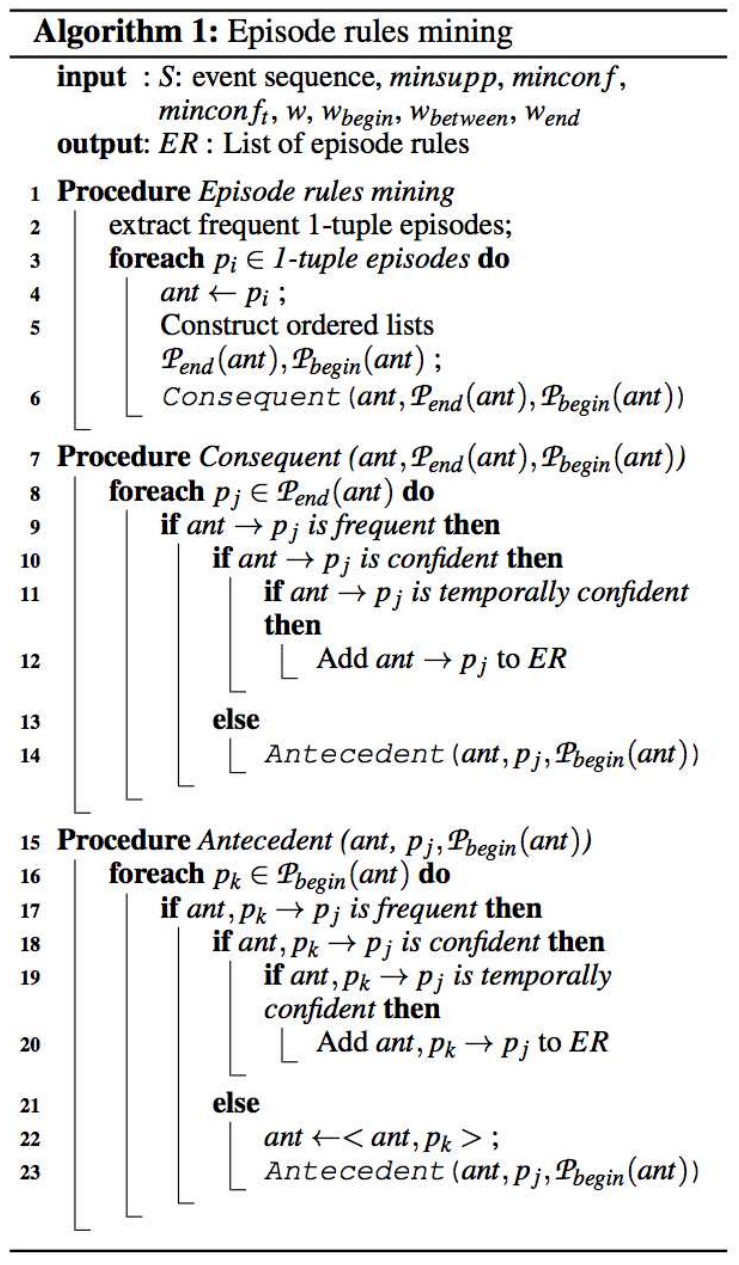

Figure 3: Episode rules mining algorithm

\section{EXPERIMENTAL RESULTS}

In this section, we evaluate our algorithm through the study of the characteristics of the episode rules formed, as well as its performance in a prediction task.

\subsection{Dataset}

The dataset we use is made up of 27,612 messages extracted from blogs about finance. Messages are an- 
Table 2: 1-tuple episodes : length and support.

\begin{tabular}{c|c|c|c|c|c|}
\hline 1-tuple episode & \multirow{2}{*}{ number(\%) } & \multicolumn{4}{|c}{ support } \\
\cline { 3 - 6 } length (\#items) & & min & max & mean & median \\
\hline 1 & $498(76.4)$ & 30 & 797 & 149.7 & 89.5 \\
2 & $147(22,5)$ & 30 & 376 & 71.5 & 55 \\
3 & $7(1)$ & 32 & 54 & 44.4 & 46 \\
\hline
\end{tabular}

notated using the Temis ${ }^{1}$ software. Each message is represented by its corresponding set of annotations (items). For example, the message: "Not only my bank propose the best, but also online banks, so how to optimize my savings? accounts in other banks? life insurance? i need more info." is annotated with the following items : \{(online banks, negative), (savings, neutral), (life insurance, neutral), (needs, neutral) $\}$, where each item is associated with an opinion degree.

In this dataset, messages are annotated with 4.8 items on average, ranging from 1 to 50 and the median is 4 . There are about 4,000 distinct annotations (items), with an average frequency of 88.5. 1,981 of these items have a frequency equal to 1 . These items will be automatically filtered out in the initialization phase of our algorithm.

\subsection{Characteristics of the resulting rules}

\subsubsection{Initialization phase}

In this phase, frequent 1-tuple episodes are extracted (1-tuple episode is made up of one or more items). We fix minsupp for these 1-tuple episodes to 30 . This phase results in 652 frequent 1-tuple episodes. Table 2 shows that the 1-tuple episodes are made up of one to three items only. 1-tuple episodes are mainly made up of one item ( $76 \%$ of them), which also have a high support (on average 149.7).

In order to study the episode rules formed, we make vary minsupp, minconf and $w_{\text {between }}$ one at a time, while fixing others.

\subsubsection{Making vary minsupp}

In Figure 4, we make vary minsupp from 10 to 35 to study the number of rules. Other parameters remain fixed: minconf $=0.4, w=40$ (with $w_{\text {begin }}=$ $20, w_{\text {between }}=10$ and $w_{\text {end }}=10$ ). As expected, the smaller minsupp, the higher the number of rules. When minsupp is fixed to 10 , the number of rules is high (about 104). Recall that the number of 1tuple episodes is only 652. However, the number of rules is dramatically decreased when minsupp is increased: only 1,200 when minsupp $=15$ and 250

\footnotetext{
${ }^{1}$ http://www.temis.com
}

when minsupp $=20$. These low values are due to the small average frequency of 1-tuple episodes (about 88 ). In addition, these rules represent a temporal dependence between the antecedent and the consequent which, in these experiments, is at least $w_{\text {between }}=10$. This may explain the low number of rules. A thorough study shows that their average confidence increases with minsupp.

\subsubsection{Making vary minconf}

We make vary minconf from 0.1 to 0.5 . Figure 5 presents the number of episode rules according to the value of minconf (minsupp $=20, w=40\left(w_{\text {begin }}=\right.$ $20, w_{\text {between }}=10$ and $\left.w_{\text {end }}=10\right)$ ). The number of rules is particularly high with mincon $f=0.2$. This is explained by the way our rules are formed. When the confidence of a rule does not exceed minconf, its antecedent is extended. Given an antecedent ant, events in $\mathcal{P}_{\text {begin }}$ (ant) (up to 652) are appended to it, resulting in a large number of candidate rules. Some of them are confident, which explains the increase of the number of rules. When minconf exceeds 0.2 , the number of rules decreases.

Table 3 presents the length of the antecedent of the rules (in number of events), according to minconf . The maximum length of an antecedent is three. Thus, our algorithm, forms rules with a small antecedent, which was one of its goals. The average length of the antecedent increases with mincon $f$ : when mincon $f=$ 0.1 , most of the rules have an antecedent of length 1 , whereas when minconf $=0.3$, most of the rules have an antecedent of length 2 . This was expected, as minimal antecedents are searched. Indeed, when a frequent rule has a confidence below minconf, its antecedent is extended, till it is confident or not frequent. So, the higher minconf $f$ the larger the antecedents. A thorough study shows that the average length of occurrence windows of the antecedents is 8 timestamps (for antecedents of length 2 and 3), which is smaller than the span of the antecedent $\left(w_{\text {begin }}\right)$. We conclude that our algorithm succeeds in forming rules with a distant consequent, a small antecedent (in length and time) and a relatively high confidence.

Table 3: Antecedent length when making vary minconf.

\begin{tabular}{c|c|c|c|c}
\hline \multirow{2}{*}{ minconf } & \multirow{2}{*}{ \#Rules } & \multicolumn{3}{|c}{ \%Rules } \\
\cline { 3 - 5 } & & ant 1 & ant 2 & ant 3 \\
\hline 0.1 & 16,850 & 57.2 & 42.84 & 0 \\
0.2 & 31,972 & 4.2 & 95.6 & 0.2 \\
0.3 & 5,072 & 0.9 & 97.5 & 1.6 \\
0.4 & 251 & 0.8 & 90.9 & 8.3 \\
0.5 & 9 & 0 & 100 & 0 \\
\hline
\end{tabular}




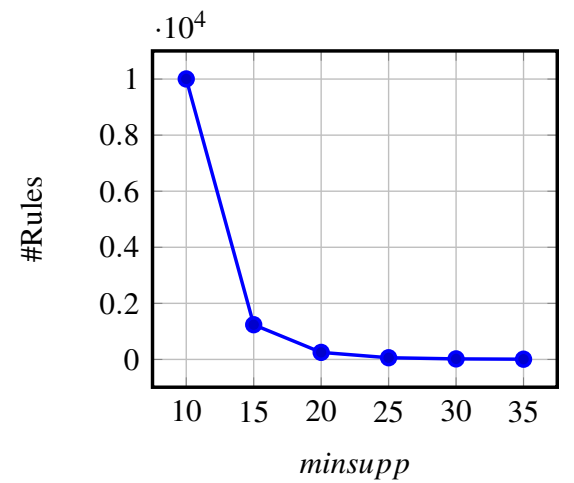

Figure 4: number of rules $\left(10^{4}\right)$ vs. minsupp

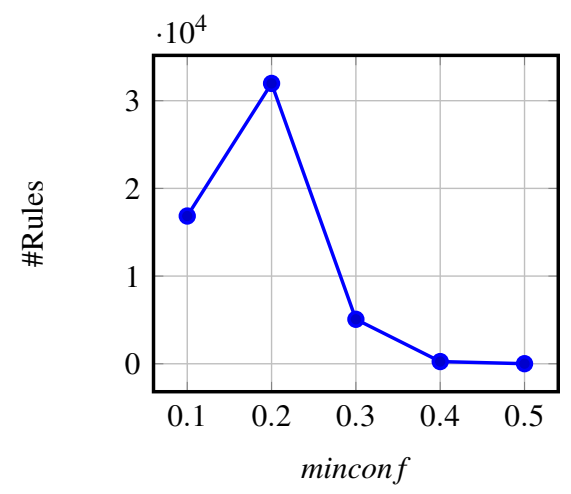

Figure 5: number of rules $\left(10^{4}\right)$ vs. minconf

\subsubsection{Making vary $w_{\text {between }}$}

We now focus on the number of rules formed, according to $w_{\text {between }}$ ( $w$ and $w_{\text {begin }}$ remain fixed), presented in Figure 6 where minsupp $=20$ and minconf $=0.4$. Two values of $w$ are studied: $w=40$ (with $w_{\text {begin }}=$ $20)$ and $w=100\left(w_{\text {begin }}=20\right)$. Notice that the cases $w_{\text {between }}=0$ represent similar cases than state of the art. We note that the larger $w_{\text {between }}$, the smaller the number of rules. Two reasons may explain this decrease. First, when $w_{\text {between }}$ increases, $w_{\text {end }}$ (the window in which the consequent is searched) decreases, as well as the number of consequents studied. Second, the larger $w_{\text {between }}$, the more distant the consequent, thus the lower the probability of having a dependence between the antecedent and the consequent. However, even with a large value of $w_{\text {between }}$, some rules are formed: 210 rules when $w_{\text {between }}=70$. We conclude that there is actually a temporal dependence between messages in blogs. When the minimal distance between the antecedent and the consequence is 50, more than 140k confident rules are formed: there is a strong dependence between messages with such a distance. For example, when $w=100$ an episode rule: (price, positive), (information, positive) $\rightarrow$ (buy, positive), means that when someone talks about the

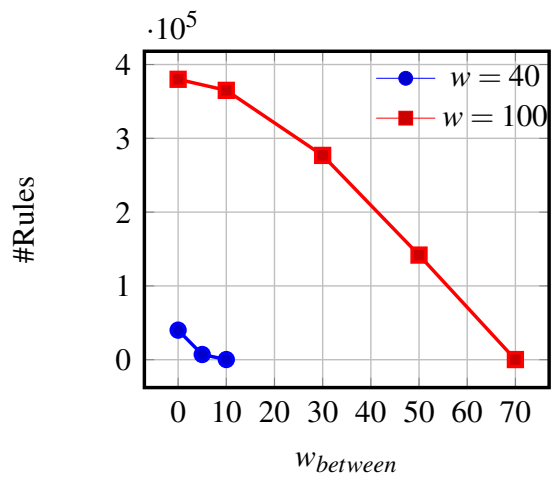

Figure 6: number of rules $\left(10^{5}\right)$ vs. $w_{\text {between }}$

price of an article then asks for information, he/she will buy this article after some time. Thus, we have time to recommend him similar articles or to propose to him a credit to buy it.

Influence of $\operatorname{mincon} f_{t}$ : In this section we study the temporal confidence of the resulting rules. Table 4 presents the evolution of the temporal confidence according to $w_{\text {between }}$. We remark that the smaller $w_{\text {between }}$, the higher the temporal confidence: the consequent does rarely occur between the antecedent and the consequent when the gap between them is small, which was expected. When $w_{\text {between }}=30$, the average temporal confidence is 0.6 , which is quite high. A thorough study shows that among $2.7 \cdot 10^{5}$ rules (see Figure 6), about 40 ones have a temporal confidence equal to 1 (the consequent never occurs in $\operatorname{Win}_{\text {between }}$ ) and 1,400 rules have a temporal confidence higher than 0.9 (the consequent appears in $W_{\text {in }}$ between in less than $10 \%$ of the cases). This shows that in this dataset there is a strong temporal dependence between events, and that some events are interdependent at a distance of 30 .

So, when exploiting the temporal confidence as a filter, a great number of rules remain.

Table 4: $w_{\text {between }}$ vs. Temporal confidence $\left(\operatorname{con} f_{t}\right)$.

\begin{tabular}{c|c|c|c|c}
\hline$w_{\text {between }}$ & min-con $f_{t}$ & max-con $f_{t}$ & mean-con $f_{t}$ & median-con $f_{t}$ \\
\hline 70 & 0 & 0.5 & 0.2 & 0.2 \\
50 & 0.2 & 0.9 & 0.4 & 0.4 \\
30 & 0.3 & 1 & 0.6 & 0.6 \\
10 & 0.2 & 1 & 0.9 & 0.9 \\
\hline
\end{tabular}

\subsection{Performance}

In this section we focus on the accuracy of the rules formed when they are used to predict events, and we perform a comparison of the rules with those of a traditional algorithm.

We evaluate the accuracy of our algorithm with 
the traditional recall and precision measures. The episode rules are trained on the first $75 \%$ messages and are tested on the $25 \%$ of messages left. Figure 7 presents the resulting precision and recall at 20. We fix minsupp $=20$, minconf $=0.4, w=100$ and $w_{\text {begin }}=20$. We make vary $w_{\text {between }}$ from 10 to 70. First of all, mention that two precision and recall values with two different values of $w_{\text {between }}$ are not directly comparable as they are not computed on the same data (the windows $W_{i n}$ end, on which they are computed vary in size). Both precision and recall curves decrease as $w_{\text {between }}$ increases. This was expected as the number of rules decreases. When $w_{\text {between }}=70$ (and $w_{\text {end }}=10$ ), both precision and recall values are quite low. This was expected as the rules aim at predicting events distant to at least 70 , in an occurrence window of length $w_{\text {end }}=10$. The prior probability of predicting events accurately is low. Let us now consider $w_{\text {between }}=30$, as in the previous section. We can see that both precision and recall values are quite high. When an event is predicted, in $37 \%$ of the cases, it actually occurs and events that occur in the sequence are predicted by our rules in $70 \%$ of the cases.

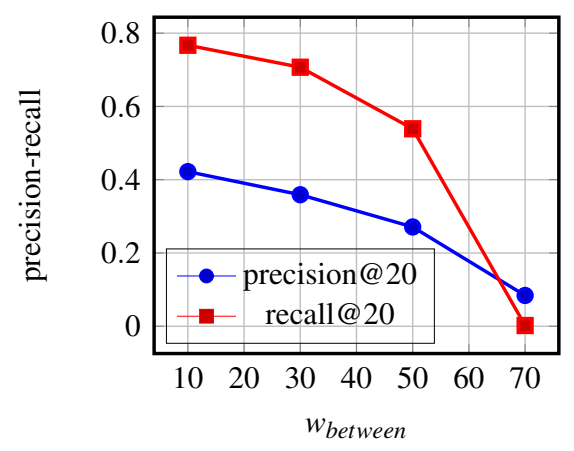

Figure 7: precision, recall vs. $w_{\text {between }}$

Comparison with Minepi: Contrary to traditional algorithms, our algorithm forces a minimum gap of length $w_{\text {between }}$ between the antecedent and the consequent of an episode rule. Our algorithm can be compared to traditional algorithms when $w_{\text {between }}=0$. Since we apply the minimal occurrence-based frequency, we choose to compare it to the well-known Minepi (Mannila et al., 1997). For minsupp $=20$, minconf $=0.4, w=40$, Minepi forms more than 136,000 episode rules, whereas our algorithm (when $w_{\text {between }}=0$ ) extracts about 40,000 episode rules (70\% less). This decrease is due to two reasons. First, the constraint about the position of consequent of the episode rules from our algorithm (in this case the distance between the antecedent and the consequent is at least 20 , even if $w_{\text {between }}=0$ ), makes the number of rules resulting from our algorithm lower (also their support is lower). Second, our algorithm aims at forming minimal rules, thus few rules with a large antecedent are formed. We remark that $25 \%$ of the rules extracted by Minepi have an antecedent larger or equal to 3 , whereas this rate is only $1.8 \%$ for our algorithm.

Here an example of an episode rule extracted by both our algorithm $\left(w_{\text {between }}=0\right)$ and by Minepi: (credit, positive), (consultant, positive) $\rightarrow$ (loan subscription, positive).

Here is a rule that has not been extracted by our algorithm, as it does not satisfy the desired characteristics of episode rule (minimal antecedent): (consultant, neutral), (interest rate, positive) $\rightarrow$ (request interest rate 0 , positive), where the antecedent occurs in 5 timetstamps and consequent occurs in the $7^{\text {th }}$ timestamp. This rule is useful in traditional cases of event prediction (prediction of close events). However, it does not fit our objective of early prediction of distant events, as the antecedent is so long both in time and in number and the consequent is too close to the antecedent.

Concerning the running time, our algorithm runs 5 times faster than Minepi when $w=100$, and 4 times faster when $w=40$. This decrease is due to two factors. The first one is related to the consequent, which is fixed at an early stage of the algorithm and which allows to filter infrequent rules early in the process. The second one is due to the fact that our algorithm mines rules with a minimal antecedent, which avoids some iterations once a confident rule is found.

\section{CONCLUSION}

In this paper, we propose an algorithm that mines episode rules, in order to predict distant events. To achieve our goal, the algorithm mines serial episode rules with distant consequent. We determine several characteristics of the episode rules formed: minimal antecedent, and a consequent temporally distant from the antecedent. A new confidence measure, the temporal confidence, is proposed to evaluate the confidence on distant consequents. Our algorithm is evaluated on an event sequence of annotated social networks messages. We show that our algorithm is efficient in extracting episode rules with the desired characteristics and in predicting distant events.

Since we use data from social networks, we aim to use multi-thread sequences. This means that we construct a sequence for each thread of messages: user messages thread, topic messages thread and discussion thread, etc. and the algorithm is run on each one. Using multi-thread sequences allows to build more di- 
verse episode rules which are all together more significant. The presence of a rule in several threads will increase its confidence.

\subsubsection{Acknowledgements.}

This research is supported by Crédit Agricole S.A.
Pasquier, N., Bastide, Y., Taouil, R., and Lakhal, L. (1999). Discovering frequent closed itemsets for association rules. In Database Theory-ICDT, pages 398-416. Springer.

Rahal, I., Ren, D., Wu, W., and Perrizo, W. (2004). Mining confident minimal rules with fixedconsequents. In 16th IEEE ICTAI 2004.

\section{REFERENCES}

Achar, A., Sastry, P., et al. (2013). Pattern-growth based frequent serial episode discovery. Data \& Knowledge Engineering, 87:91-108.

Agrawal, R., Imieliński, T., and Swami, A. (1993). Mining association rules between sets of items in large databases. In ACM SIGMOD Record, volume 22, pages 207-216. ACM.

Cho, C.-W., Wu, Y.-H., Yen, S.-J., Zheng, Y., and Chen, A. L. (2011). On-line rule matching for event prediction. The VLDB Journal, 20(3):303334.

Gan, M. and Dai, H. (2011). Fast mining of nonderivable episode rules in complex sequences. In Modeling Decision for Artificial Intelligence. Springer.

Huang, K.-Y. and Chang, C.-H. (2008). Efficient mining of frequent episodes from complex sequences. Information Systems, 33(1):96-114.

Laxman, S., Sastry, P., and Unnikrishnan, K. (2007). A fast algorithm for finding frequent episodes in event streams. In 13th ACM SIGKDD. ACM.

Laxman, S. and Sastry, P. S. (2006). A survey of temporal data mining. Sadhana, 31(2).

Luo, J. and Bridges, S. M. (2000). Mining fuzzy association rules and fuzzy frequency episodes for intrusion detection. Int. J. of Intelligent Systems, 15(8):687-703.

Mannila, H., Toivonen, H., and Verkamo, A. I. (1997). Discovery of frequent episodes in event sequences. Data Mining and Knowl. Discovery, 1(3):259-289.

Méger, N. and Rigotti, C. (2004). Constraint-based mining of episode rules and optimal window sizes. In PKDD 2004, pages 313-324. Springer.

Neeraj, S. and Swati, L. S. (2012). Overview of nonredundant association rule mining. Research Journal of Recent Sciences ISSN, 2277:2502.

$\mathrm{Ng}$, A. and Fu, A. W.-C. (2003). Mining frequent episodes for relating financial events and stock trends. In Advances in Knowledge Discovery and Data Mining, pages 27-39. Springer. 